\title{
Distal upper limb disorders and ergonomics of VDU work: a review of the epidemiological evidence
}

\author{
Kaj Bo Veiersted and Morten Wærsted \\ National Institute of Occupational Health, P.O.Box 8149 Dep, N-0033 Oslo, Norway \\ Telephone: 23195100 Telefax: 23195205 E-mail: Bo.Veiersted@stami.no
}

\begin{abstract}
A review is presented of epidemiological peer-reviewed publications that relate the mechanical exposure of the arm in work with visual display units (VDU) to musculoskeletal disorders of the upper limb. The twenty included studies were divided into those with (1-7) and without (8-21) use of physical examination in the definition of cases. The main conclusion of this review is the documentation of evidence for the VDU work per se, none or insufficient lower arm support and non-neutral positions of the wrist as predictors of distal upper limb disorders (DULDs). These findings are based solely on studies that use physical examination or objective signs to define the DULDs, and the conclusions are not changed after inclusion of studies based only on self-reported symptoms. When the last category of studies is included as documentation in the review, the amount of VDU work (and an exposuredose/effect relationship) will also be documented as predictors. The referred studies indicated that not only the VDU work per se, but also (and in particular) VDU work for more than 20 hours per week combined with limited opportunity for rest breaks and without lower arm support is a predictor of DULDs. Another conclusion is that critical reviews may benefit from use of both strict criteria and less strict criteria for inclusion of studies, to optimize the conclusions drawn from existing documentation.
\end{abstract}

\section{INTRODUCTION}

Use of personal/terminal computers or video display units (VDUs) has been focused upon during the last decades in studies of several different work-related predictors of musculoskeletal disorders (22-27). Most reviews do not use critical inclusion criteria to extract high quality documentation, and since the quality of the existing epidemiological literature on this topic has been criticized, it has been difficult to draw firm conclusions (27). However, some recent critical reviews have concluded with an existing strong evidence for a causal relationship between static muscular activity of the neck muscles and musculoskeletal disorders in the neck region $(28,29)$. A direct causal relationship between the use of VDU and hand or wrist disorders is also proposed in a recent review (30).

Many epidemiological studies only use selfreported symptoms of musculoskeletal disorders. This may invalidate the outcome estimates since existing pain symptoms may be exaggerated/provoked by heavy or unsatisfactory work postures and not caused by them. Self-reported symptoms may sometimes be evaluated by the same questionnaire as exposure estimates, which then may cause an increased likelihood to report mechanical exposure among subjects with symptoms (differential misclassification, information bias). It is therefore of interest to evaluate if the inclusion of studies that use self-reported symptoms change conclusion based on studies using physical examination for their outcome definition.
The background for this review is an ongoing work in a Nordic expert committee that evaluates the possibilities of a criteria document on mechanical exposures. The aim of that work is to review the literature dealing with the relationship between mechanical exposure of the upper limb at work and musculoskeletal disorders $(31,32)$, and evaluate the possibility to make standards for mechanical exposures. The present paper extracts the documentation on VDU work and adds the supposed best of the studies not included by the review of the mentioned group.

The main aim of this paper is to review the evidence for specific ergonomic predictors (mechanical exposures) of musculoskeletal disorders in the distal upper limb (distal to the shoulder). A second aim is to compare conclusions of the work-relatedness of distal upper limb disorders (DULDs) in VDU work based on studies with and without the use of physical examination in the case definition.

\section{METHODS AND LITERATURE SEARCH}

A literature search was performed to find peerreviewed publications in English relating mechanical exposure of manual load to symptoms or diagnoses of the distal upper limb. Reports, abstracts and proceedings were not included.

Distal upper limb was here defined as the arm distal to the shoulder or the insertion of m. deltoideus on humerus. Musculoskeletal diagnoses (or Distal Upper Limb Disorders, DULDs) of this region are mainly: 
the lateral epicondylitis, the tenosynovitis/tendovaginitis («tendinitis») of the forearm/wrist and the carpal tunnel syndrome. These are the disorders included as outcome variables in the present review. Other musculoskeletal disorders of this region may also be related to mechanical exposure at work but these are scarcely investigated and will not be reviewed here.

The period covered by the search was between 1966 and 1996 in the bases of MEDLINE, NIOSHTIC, BIOSIS PREVIEWS and EMBASE. The period 1996-1998 was covered by a search in the MEDLINE and NIOSH-TIC bases only. The search words included names of body parts in the distal upper limb, diagnoses and job titles, specific exposures (e.g. VDU/VDT) and generic risk factors (e.g. use of high force or repetivity).

The criteria for selection of studies were: 1) comparison between exposed and unexposed groups (or a single population with internal exposure contrast), 2) use of individual exposure estimates (exposurecharacteristics defined for each subject) and 3) symptoms or diagnoses specified for distal upper limb (e.g. excluding studies with outcomes that joins disorders or symptoms in the arm, shoulder and neck regions).

The exposure of interest for this review was the work with (or use of) VDU or VDT (visual/video display terminals). In some studies it is described as keyboard operators. The main focus is on the mechanical part of the exposure on the upper limb and the literature is included only if it to at least some degree evaluated the VDU work itself. This means that the studies have to analyze the effect on outcome either of VDU work per se or the amount of it (the two first factors listed in table 1). The literature examining VDU work was selected and then divided into studies with or without use of a physical examination or objective signs for the case definitions.

The quality of the studies that used physical examination or objective signs for case definition were assessed by the following items: 1) exposure assessment, 2) case definition (e.g. blinded diagnosis), 3) choice of control group or internal contrast, 4) response rate, 5) secondary subject selection, 6) adjustment for confounders and 7) external validity (including age of the study).

\section{RESULTS OF REVIEW}

Twenty studies were included in the review. Six studies (1-7), that were described in 7 papers, used physical examination or objective signs for the case definition and is below designated as «primary» studies. In the remaining fourteen studies (8-21), constituting the «secondary» studies, only selfreported symptoms were used for the case definitions (see Table 1). The table shows the documented relationships between certain exposures and one or several of the investigated upper limb disorders.

The results and the quality of the six studies that used a physical examination or an objective sign are initially commented upon, since these are the main sources of evidence because of the independent documentation of outcome. The rest of the included studies are commented upon in the next section where the risk factors are listed. The evidence for a certain risk factor is first based on the «primary» studies and then related to the «secondary» studies.

\section{The studies using physical examination or objective signs}

In one of the best performed studies, Bergquist et al, 1995 (1) divided office workers in two groups, those with more than 5 hours VDU work per week $(\mathrm{h} / \mathrm{w})$ (exposed, $\mathrm{n}=261$ ) and those with no or less than $5 \mathrm{~h} / \mathrm{w}$ of VDU based tasks (controls, $n=61$ ). No difference in the prevalence of arm and hand diagnoses was found between these two groups. Relatively higher levels of muscle pain complaints were reported in a subgroup that worked more than $20 \mathrm{~h} / \mathrm{w}$ with VDU work, but only when combined with limited rest opportunity and non-use of lower arm support (odds ratio: 4.6; CI 1.2-17.9). Univariate and multivariate analyses were performed on the same data set (2). Associations in univariate tests were here found between arm/hand diagnoses and lack of lower arm support as well as use of high profile keyboards. None of these individual ergonomic factors stayed significant after a multivariate analysis controlling for e.g. age, children and smoking. However, the combination of no use of lower arm support and limited opportunities for rest breaks showed an odds ratio of 10.1 (CI 2.4-43.2) for arm/hand diagnoses.

Comments: The exposure was evaluated with ergonomic measurements, but only ergonomic data from the questionnaire turned out to be significant in multivariate analyses. Case definitions were made by physiotherapists, but it is not clear if they were blinded to exposure status. The control group may be «diluted» by some low-level exposed VDU workers, eventually making the contrast low between VDU and «nonVDU» workers. With higher contrast, i.e. «non-VDU» workers compared to workers using VDU for more than $20 \mathrm{~h} / \mathrm{w}$, some significant findings were found. The study had high response rate, adjusted for confounders and commented upon secondary subject selection (a healthy worker effect may have occurred for «hand problems»). The external validity was high for this study, performed in start and mid-nineties with subjects representing a variety of companies. In summary, a well performed study but the use of low contrast between exposed and non-exposed may hide positive associations.

In a cross-sectional study by Feraz et al, 1995 (3)130 keyboard operators and 138 office workers performing mostly other tasks were interviewed and symptomatic subjects were examined by a rheumatologist. The data entry operators were significantly more likely to have «tendinitis» of the wrist and hands. Several risk factors were assessed, but only in relation to upper extremity 
Table 1. Mechanical exposures $(A+B)$ and other factors $(C+D)$ as possible predictors of distal upper limb disorders in VDU work.

\begin{tabular}{|c|c|c|c|c|c|c|c|c|c|c|c|c|c|c|c|c|c|c|c|c|c|}
\hline & Predictor studied & 1,2 & 3 & 4 & 5 & 6 & 7 & 8 & 9 & 10 & 11 & 12 & 13 & 14 & 15 & 16 & 17 & 18 & 19 & 20 & 21 \\
\hline \multirow{7}{*}{ A } & VDU work per se & & $\mathrm{CS}$ & & $\mathrm{CS}$ & $\mathrm{S}$ & $\mathrm{C}$ & & & $\mathrm{S}$ & & & & & & $\mathrm{S}$ & & & & $\mathrm{S}$ & \\
\hline & Amount of VDU work & $\mathrm{C}^{1}$ & & & & & & & $\mathrm{~S}^{2}$ & $\mathrm{~S}^{2}$ & $\mathrm{~S}^{2}$ & & & $S^{2}$ & & & & & & & \\
\hline & Length of employment & & & & & & & & & & & & & & $\mathrm{S}$ & & & & & & \\
\hline & Extensive overtime & $\mathrm{S}$ & & & & & & & & & & & & & & & & & & & \\
\hline & Insufficient rest breaks & $\mathrm{C}^{1}$ & & & & & & & & & & $\mathrm{~S}^{3}$ & & & & & & & & & \\
\hline & Keystroke rate & & & & & & & & & & & & & & & & & & & & \\
\hline & Amount of computer mouse use & & & & & & & $\mathrm{S}$ & & & & & $\mathrm{S}$ & & & & & & & & \\
\hline \multirow{5}{*}{$\mathrm{B}$} & Unsatisfactory work posture / work place & & & & & & & & & & & & & & & & & $\mathrm{S}$ & & & \\
\hline & Keyboard height (with reference to elbow) & $\mathrm{CS}^{5}$ & & & $\mathrm{C}^{5}$ & & & & & & $\mathrm{~S}^{4}$ & & & & & & & & $\mathrm{~S}$ & & \\
\hline & None/insufficient use of lower arm support & $\mathrm{C}^{1}$ & & & $\mathrm{C}$ & & & & & & & & & & & & & & & & \\
\hline & Non-optimal mouse position & & & & & & & & & & & & $\mathrm{S}$ & & & & & & & & \\
\hline & Non-neutral position of wrist/hand & $\mathrm{S}$ & & & $\mathrm{C}$ & & & & & & & & & & & & & & $\mathrm{S}$ & & \\
\hline $\mathrm{C}$ & Psychological, social or organizational factors & $\mathrm{C}$ & & $\mathrm{C}$ & & & & & $\mathrm{S}$ & & $\mathrm{S}$ & $\mathrm{S}^{3}$ & & & $\mathrm{~S}$ & & & & & & \\
\hline \multirow{3}{*}{$\mathrm{D}$} & Gender & $\mathrm{C}$ & & & & & & & $\mathrm{S}$ & $\mathrm{S}$ & & & $\mathrm{S}$ & $\mathrm{S}$ & & & & & & & $\mathrm{S}$ \\
\hline & Age & $\mathrm{C}$ & & & & & & & & & & & $\mathrm{S}$ & & & & & & & & \\
\hline & Smoking & $\mathrm{C}$ & & & & & & & & & & & & & & & & & & & \\
\hline
\end{tabular}

${ }^{1}$ Combination of amount of VDU work, limited rest break opportunities and insufficient use of lower arm support.

${ }_{3}^{2}$ Parameter showing an exposure-dose/effect relationship.

${ }^{3}$ The study used clinical finding and/or subjective pain report to classify for the outcome measure.

${ }^{4}$ Combined with low decision latitude and better supervisor relations.

${ }^{5}$ Negative association.

1-7: Six studies where a diagnosis of distal upper extremity disorder is based on physical examination or objective signs.

8-20: Fourteen studies with an outcome classificaton based on self report measures.

Open square: Indicates that the predictor was addressed in the study.

Shaded square: Indicates that the predictor was not studied (or not reported) in relation to distal upper limb disorders.

C: The study identified a positive association between predictor and a clinical diagnosis or objective sign of distal upper limb disorder.

S: The study identified a positive association between predictor and self-reported distal upper limb pain.

musculoskeletal disorders as a combined group, including shoulder and neck disorders.

Comments: Exposure analyses were attempted but rejected due to «bad data». The control group comprised workers with no or little use of keyboard with no further specification. Seventy per cent of the keyboard operators were women compared to only 56 per cent of the office workers in the control group. Symptoms reported in the interview and/or findings in a «brief» screening by a physiotherapist were criteria for a full investigation by a «blinded» rheumatologist. The response rate was satisfactory (ca. $85 \%$ ), and the same concerns the external validity (two firms examined possibly in the beginning of the nineties - dates for data collection not mentioned). Secondary subject selection was not discussed by the authors. The analyses did not control for age, gender or length of employment, but except for distribution of gender, the two groups seems to be comparable. In summary, this study has some methodological deficiencies, among others were differences in gender distribution between groups and that not all subjects were clinically examined, but the main findings are consistent with other studies.

In a cross-sectional study by Hales et al, 1994 (4) the relationship between workplace factors and workrelated musculoskeletal disorders of the upper extremity were assessed for 533 VDU users with multiple logistic regression models generated separately for four areas (neck, shoulder, elbow and hand/wrist). All workers used VDU for 6 or more hours per day and could by job title be placed in five separate job categories. In the analysis none of the risk factors described in terms with close association to physical work load (such as number of keystrokes per day, hours per day spent at the VDU, number of breaks per day) showed a significant relation to distal upper limb disorders, whereas the more psychosocial formulated risk factors «routine work lacking decision-making opportunities» and «high information processing demands» gave significantly increased odd ratios for elbow and hand/wrist disorders, respectively.

Comments: The exposure for different categories of VDU workers were rather well described and all subjects had a blinded clinical examination with well defined criteria. A particular control group was not used, but internal contrast may have occurred in the different job categories. In summary, high response rate $(93 \%)$, adjustment for confounders in multiple regression models and high external validity due to a variety of jobs under study and recent data collection (possible start of nineties - not specified in the paper) suggest this as a high quality study.

Hünting et al, 1981 (5) found that both data entry workers $(n=53)$ and typewriters $(n=78)$ had significantly increased risk of pain induced by isometric contrac- 
tions of the forearm (most resemblance with «tendinitis», eventually epicondylitis) compared to workers with a diversity of office tasks $(n=55)$ or to workers with conversational VDU-tasks $(n=109)$. Ulnar deviation above $20^{\circ}$ during keying was a risk factor for arm symptoms and signs. A frequent use of support for arms and hands was correlated to a lower incidence of pain in the arm.

Comments: The exposure evaluation was very well performed in this study from «the early time» of VDU work. This concerns both measurements and questions on work technique. The analyses did not include control for confounders, mean age was rather similar in the four groups but the gender distribution was not. This may have biased the conclusion on the effect of data entry work per se since there was an overwhelming majority of women in that group compared to control groups. In summary, this is an old study with insufficient control for confounders which questions the external validity of effects of data entry work per se, but, on the other hand, the effect of the more precise described work exposures seems to be valid also in the late nineties.

In a Finnish study by Kukkonen et al, 1983 (6) the health of sixty female data entry operators was assessed through a questionnaire and a standardized physical functional examination of the neck and upper limbs. The assessment was done before and after a six month intervention period. Another group of data entry operators $(n=44)$ as well as a group of women $(n=57)$ performing other office tasks were included as reference groups. At the first health examination, the data entry operators (the study group and the first reference group) reported significantly more numbness in the elbows (as well as neck, shoulder and lower limb symptoms) compared to the second reference group of office workers. In the physical examination, only the neck symptoms was different between data entry and office workers.

Comments: This is also an older study analyzing data entry work in the beginning of the eighties, performing an elaborated ergonomic survey but not using it in the analyses. Despite this paper fulfilling the inclusion criteria, the analysis of VDU per se is from almost 20 years ago, and the lack of more precise measurements of mechanical exposures, reduces the external validity of the study in relation to VDU work in the late nineties.

In a more recent cross-sectional study by Murata et al, 1996 (7) they reported a reduced nerve conduction velocity as an objective sign of carpal tunnel syndrome (CTS) as a mean for 27 female VDU users (data entry work about 6 hours per day) compared to 19 female non-users. No other evaluations of work exposure was made. As a group the VDU users complained more of CTS symptoms but the complaints were not associated to reduced nerve conduction. No subjects had previously been pregnant or have had neurological or endocrinological disorders or been exposed to neurotoxic substances.

Comments: The exposure estimation was rather coarse but made a contrast between users and nonusers. However, exposures of the wrist was not described for the control group, except for «sometimes use of word processor». Only reduced nerve conduction was evaluated as a sign of clinical or subclinical CTS. The results were not adjusted for confounding factors but seemed very well matched and were comparable with respect to age, health status and skin temperature during test.

\section{The effect of predictors}

The results of the review are summarized in Table 1, showing the six studies that include a physical examination to the left, and the other fourteen studies to the right. The risk factors are grouped in four categories (letters refer to labeling in Table 1): A) VDU work and time distribution of exposure, B) Posture and work place design, C) Psychological, social and organizational factors, and D) Individual factors. Because of the inclusion criteria of this review none of the studies in Table 1 lack information on both the first two predictors. The rest of the predictors are more or less investigated in the included literature, but when analyzed they are presented in the table as white boxes with or without letters indicating whether or not a positive association was found.

Each mechanical exposure (predictor or risk factor) is first described, in the review of the results below, and then the main impression from the table is presented. This includes a differentiation between information from «primary» and «secondary» studies.

\section{VDU work and time distribution of exposure}

(Table 1, predictor category A)

The exposure of VDU work (or VDU use) per se means simply if the exposure is present or not. It includes different (or all) aspects of the work as a keyboard operator. This means that all mechanical, psychological, social and organizational factors that may differentiate between VDU work and exposures of a control group may influence the result.

Nearly all the «primary» studies have included an assessment of risk from VDU work per se. Five of the studies with physical examination included this analysis, and three of them found a significantly increased risk for clinical findings (marked with «C» in Table 1). For two of these studies the clinical finding was «tendinitis» $(3,5)$ and for one it was objective signs of reduced nerve conduction velocity (7). The study that used physical examination but only found significant result for self-reported arm pain (marked with «S» in Table 1) described it as «numbness in the elbows», a possible symptom of epicondylitis (6). Perhaps the best and most comprehensive study $(1,2)$ found no relationship between VDU work per se and neither complaints nor diagnoses in the arm and wrist region. 
Despite this, one may stress that four out of five «primary» studies looking for it also found a positive association between VDU work per se and DULDs. «Secondary» studies only include subjective reporting for case definition (right hand part of Table 1), and two out of the six studies that examined it were able to identify VDU use per se as a risk factor for wrist, hand or finger symptoms $(10,16)$. One study found a negative association to «pain down arm» (20), and the remaining three found no association of VDU work to pain symptoms in the arm. The «secondary» studies do not clearly confirm the findings of the «primary» studies for VDU work per se.

The amount of VDU work are in the included studies mostly defined as this kind of work for hours per day or per week. Two of the «primary» studies assessed the amount of VDU use per workday, and one showed a significantly increased risk but only when the increased VDU use were combined with limited rest breaks and non-use of lower arm support $(1,2)$. Twelve of the fourteen "secondary» studies have data on the amount of VDU use per workday. Four of these studies $(9-11,14)$ identified an exposure-dose/ effect relationship between VDU work and symptoms. The symptoms concerned pain or numbness in wrist, hand or fingers and, for Knave et al, musculoskeletal symptoms in general. The «critical level» of VDU work per day has been shown to be 4 hours (or $20 \mathrm{~h} / \mathrm{w}$ ) $(1,2)$, and 6 hours $(9)$. A cumulative incidence increase of $0.32 \%$ per hour of increased weekly VDU work was shown in one study (10). The main impression is that the "primary» studies do not give any sufficient documentation on exposure-dose/effect relationship. However, the «secondary» studies show a rather consistent association between the amount of VDU work and symptoms.

The length of employment with VDU-based work may be conceived as a proxy for dose of VDU work. It is always described in years. This variable was evaluated in three of the «primary» studies and three of the studies based on subjective reports. Only one of the latter studies (15) identified a relationship to distal upper limb pain. Of other indices of VDU work dose, extensive overtime was evaluated in two "primary» studies and one «secondary» study, but only subjective measures showed an association to overtime work in one of the «primary» studies $(1,2)$. In that study also the reporting of insufficient rest breaks showed a relationship to clinical signs of distal upper limb disorders, and similarly one (12) out of three «secondary» studies found such an association (Table 1). Keystroke rate (a measurement of work speed) was available for assessment in a subsample of 174 workers (out of 533) in one study (4), but this measure showed no relationship to clinical findings in that study. Amount of computer mouse use (hours per day) in relation to disorders or complaints in the distal upper limb has been evaluated in two studies $(8,13)$ and both showed a positive association to self-reported arm pain.

\section{Posture and work place design}

(Table 1, predictor category B)

Unsatisfactory work posture and work place design is a predictor difficult to define, and is not evaluated as such in the «primary» studies. The «secondary» studies use very different definitions and sources of information for this possible predictor. Two studies used self-reported assessment of this predictor $(12,21)$, one used trained observers (11) and one performed videorecording of a subsample (18) to evaluate the work postures. The three former did not find any positive association, the latter was the only study that found an association between this predictor and measured postural factors, as for example high position of screen and non-adjustable keyboard.

The height of the keyboard is mostly described as the relative height to the elbow $(1,2,5)$ but also the height of the keyboard itself. The height of the keyboard itself was positively associated to clinical findings $(5)$. The two «primary» studies $(1,2,5)$ that investigated the effect of position of the keyboard in relation to elbow found a negative association, i.e. increased risk for symptoms and signs of DULDs with lowering of the keyboard. One of the «secondary» studies (19) showed a significant effect of high positioned keyboard on symptoms of arm discomfort. Another study (11) (see Table 1, including footnote 4) only showed an increased risk for symptoms if the increased height was combined with low decision latitude and «better» supervisor relations. The third «secondary» study (13) found no effect of the height of keyboard.

None or insufficient use of lower arm support is mostly defined as a lack of support for the whole forearm during work. However, in the study by Hünting et al (5) «hands and arms frequently supported» was negatively associated to arm pain. Bergquist et al $(1,2)$ found a strong indication that non-use of lower arm support alone was a predictor of arm/hand diagnoses (odds ratio 2.7; CI 0.9-8.3) but was a significant predictor together with 20 hours per week of VDU work and limited rest break opportunity. The only «secondary» study that investigated this predictor could not document any significant effect on pain in the forearm and hands (8), but instead this was found for neck pain.

One study evaluated a non-optimal computer mouse position (13) and found an increased the risk in that situation for reporting musculoskeletal symptoms in elbow, wrist and hand/fingers.

The last risk factor in category B (Table 1) evaluated in the two «primary» studies, was a non-neutral position of the wrist and hand. This was significantly related to subjective reports in one study $(1,2)$, and clinical findings in the other study (5). The latter study found the increased risk especially for ulnar deviation above $20^{\circ}$. This kind of predictor was also evaluated in the three studies with only subjective outcome mea- 
sures, showing a significant positive association in one of them, especially for right-hand extension (19).

Some other risk factors concerned with the ergonomic lay out (i.e. the adjustability of the workstation and the keyboard) or a more general statement of an unsatisfactory work posture or work station lay out was assessed in three studies with a subjective outcome measure, giving a significant relationship in one of them (18), see first line under category B in Table 1.

\section{Psychological, social or organizational factors}

(Table 1, predictor category C)

Some of the studies reviewed in this paper have assessed work-related risk factors that is not readily grouped together with category A or B of Table 1, but that are pinpointing psychological, social or organizational aspects of the work, the often called «psychosocial» factors. (It may be argued, as we will comment upon in the Discussion section, that also several of the risk factor here put in category $\mathrm{A}$, can be perceived as being measures of psychological, social or organizational aspects of the VDU work.) In the studies using such measures, one or more of these variables have shown a relationship to distal upper limb problems in all but one study (Table 1).

\section{Individual factors (Table 1, predictor category D)}

Of individual factors that often are regarded as confounders, the effect of gender was assessed in two «primary» and five «secondary» studies (Table 1). Females were observed to have an increased occurrence of DULDs and symptoms in all studies but one (4). Age was observed to have an influence in one of two «primary» studies and in one of three «secondary» studies (Table 1). Smoking habits was assessed in two studies, one study $(1,2)$ found a positive association to clinical findings, the other did not (14).

\section{DISCUSSION}

This review of predictors of distal upper limb disorders (DULDs) includes an evaluation of «primary» and «secondary» studies as sources of information. All included studies fulfilled several inclusion criteria, but «primary» studies used a physical examination for definition of cases, «secondary» studies only selfreported symptoms of the distal upper limb. By use of only «primary» studies VDU work per se, none or insufficient lower arm support and non-neutral positions of the wrist and hand seems to be predictors of DULDs. The amount of VDU work and the exposuredose/effect relationship are further information added if «secondary» studies also are included as basis for the review.

Many epidemiological studies have been performed of the effect of VDU-work on musculoskeletal disorders, but only a few passed the selection criteria of this review. These criteria were; use of a control group or contrasts within exposed, individual exposure estimates and symptoms or diagnoses specified for the distal upper limb. The studies were classified as «primary» when clinical examination was performed for case definition, thereby possibly excluding some quality papers, but on the other hand also ensuring an inclusion of studies with minimal methodological deficiencies. These papers were carefully evaluated for different quality criteria independent of inclusion criteria and only one was evaluated as having insufficient quality, mainly due to low external validity (6). The effect of reducing the strict criteria by also accepting studies with only self-reported symptoms as case definition may then reintroduce the quality paper lost in the strict selection. It seems that inclusion of «secondary» studies do not change or contradict the findings based on the «primary» studies alone. The quality of «secondary» studies was not penetrated in this review, it was enough that they passed the inclusion criteria. However, there may have occurred high quality studies not using clinical examination among them, as it occurred studies with lower quality among the «primary» studies.

The review finds evidence for a well documented association between VDU work per se and DULDs. The «primary» studies $(3,5-7)$ include sufficient evidence independent of the «secondary» studies $(10,16)$. The latter group of studies do not contribute with essential documentation, since they are few and inconclusive. The conclusion may have been more uncertain if it was based equally weighted on both «primary» and "secondary» studies. A reason why the comprehensive study by Bergquist et al $(1,2)$ do not find any association may be a low contrast level between exposed and unexposed. On the other hand, if a conclusion should be based alone on the "primary» studies $(1,2)$ of the effect of the amount of VDU work, the strong and consistent findings of the "secondary» studies $(9-11,14)$ would be lost.

The significance of «VDU work per se» as a mechanical exposure indicator is questionable. As mentioned previously, VDU work includes mechanical, psychological, social and organizational components (the latter three may be called «psychosocial») and it may therefore be difficult to extract the effects of mechanical factors. This is only possible if the control group is exposed to the same amount of «psychosocial» factors, which is not controlled for in the cited studies. Hence, «VDU work per se» is in this review an indicator of «total» exposure.

A possible effect on DULDs of length of employment, use of extensive overtime, insufficient rest breaks alone and the keystroke rate is not possible to conclude on based on the present review. The effect of amount of computer mouse use is also difficult to conclude upon. None of the «primary» studies analyzed this exposure. All the «secondary» studies that examine this question documents an association between 
computer mouse use and arm pain. However, there are only two included studies in the review so it may be concluded that only some evidence exists for a causal relationship between mouse use and arm pain $(8,13)$.

The documentation for several posture variables are inconclusive. This concerns work postures, work place design and non-optimal computer mouse position. The evidence for the effect of height of the keyboard is diverging. The «primary» studies point out that a low position is a predictor of DULDs $(1,2,5)$, but the opposite is found in one of the «secondary» studies (19). In this case the latter seems to be the most plausible association. The height of the keyboard is largely no problem in modern work stations, but the upwards tilting of the back of the keyboards (using the small legs) may result in a deleterious extension of the wrist. The beneficial effect of the use of lower arm support seems to be well documented $(1,2,5)$, although almost no documentation from the «secondary» studies exists. Non-neutral positions of the wrist seems also to be a well documented predictor of symptoms and signs of DULDs $(1,2,5,19)$. This evidence is supported by several laboratory studies by for example Rempel et al (33).

«Psychosocial» factors have been associated to DULDs in several studies. These factors are outside the scope of this review. However, some of the mechanical factors analyzed in this review may also be evaluated as partly «psychosocial». This concerns «amount of VDU work» (work load, eventually leading to time pressure), «overtime work» and «amount of rest breaks» (both organizational factors). In this review they have been looked upon as time dependent amount of work, eventually also the distribution between work and rest. It may also be argued that «psychosocial» risk indicators may reflect differences in mechanical exposure, in particular if the mechanical work load is only crudely documented or not documented at all. Thus, the relationship between and separation of mechanical factors on one side and psychological, social and organizational factors on the other side, should be clarified in future research.

The evidence for the relation between mechanical exposure in VDU work and DULDs has mainly too low precision to be used as background for scientifically (evidence) based standards. For example subjectively assessed insufficient rest breaks is not possible to use in this connection. However, despite insufficient evidence at the moment, a few mechanical exposures may be considered. It seems reasonable that the amount of «intensive» VDU work should not exceed 4 hours per day. Support for the lower arms, preferable on the table, should be accessible and the wrist should be kept in neutral position during VDU work.

The presented way of performing a review may be a useful method to make an evidence based review and make it open to important information in weaker documentation at the same time.

\section{CONCLUSIONS}

The main conclusion of this review is the documentation of evidence for the VDU work per se, none or insufficient lower arm support and non-neutral positions of the wrist as predictors of distal upper limb disorders (DULDs). These findings are based solely on studies that use physical examination or objective signs to define the DULDs (strict criteria). The amount of VDU work (including the exposure-dose/effect relationship) are also found to be predictors of DULDs when the studies with only self-reported symptoms are included as documentation in the review.

The scientific documentation for the development of a standard for mechanical exposure of the upper limb during VDU work is insufficient.

The inclusion of studies based on less strict criteria, do not seem to affect the conclusions of the review. The predictors found in the studies that were included with less strict criteria may supplement the conclusion if they are strong and consistent.

\section{REFERENCES}

1. Bergqvist U, Wolgast E, Nilsson B, Voss M. The influence of VDT work on musculoskeletal disorders. Ergonomics 1995; 38: 754-762.

2. Bergqvist U, Wolgast E, Nilsson B, Voss M. Musculoskeletal disorders among visual display terminal workers: individual, ergonomic, and work organizational factors. Ergonomics 1995; 4: 763-776.

3. Ferraz MB, Frumkin H, Helfenstein M, Gianeschini C, Atra E. Upper-extremity Musculoskeletal disorders in keyboard operators in Brazil: A cross-sectional study. Int J Occup Environ Health 1995; 1: 239-244.

4. Hales TR, Sauter SL, Peterson MR, et al. Musculoskeletal disorders among visual display terminal users in a telecommunications company. Ergonomics 1994; 37: 1603-1621.

5. Hünting W, Läubli T, Grandjean E. Postural and visual loads at VDT workplaces. I. Constrained postures. Ergonomics 1981; 24: 917-931.

6. Kukkonen R, Luopajärvi T, Riihimäki V. Prevention of fatigue amongst data entry operators. In: Kvålseth, ed. Ergonomics of Workstation design. London: Taylor \& Francis, 1983: 28-34.

7. Murata K, Araki S, Okajima F, Saito Y. Subclinical impairment in the median nerve across the carpal tunnel among female VDT operators. Int Arch Occup Environ Health 1996; 68: 75-79. 
8. Aarås A, Horgen G, Bjørset H-H, Ro O, Thoresen M. Musculoskeletal, visual and psychosocial stress in VDU operators before and after multidisciplinary ergonomic interventions. Appl Ergonomics 1998; 29: 335-354.

9. Bernard B, Sauter S, Fine L, Petersen M, Hales T. Job task and psychosocial risk factors for work-related musculoskeletal disorders among newspaper employees. Scand J Work Environ Health 1994; 20: 417-426.

10. Bergqvist U, Knave B, Voss M, Wibom R. A longitudinal study of VDT work and health. Int J HumComputer Interact 1992; 4: 197-219.

11. Faucett J, Rempel D. VDT-related musculoskeletal symptoms: Interactions between work posture and psychosocial work factors. Am J Ind Med 1994; 26: 597-612.

12. Ferreira M, Conceicão GMS, Salvida PHN. Work organization is significantly associated with upper extremities musculoskeletal disorders among employees engaged in interactive computer-telephone tasks of an international bank susidiary in São Paulo, Brazil. Am J Ind Med 1997; 31: 468-473.

13. Karlqvist LK, Hagberg M, Köster M, Wenemark M, Ånell R. Musculoskeletal symptoms among computerassisted design (CAD) operators and evaluation of a self-assessment questionnaire. Int J Occup Environ Health 1996; 2: 185-194.

14. Knave BG, Wibom RI, Voss M, Hedström LD, Bergqvist UOV. Work with video display terminals among office employees: I. Subjective symptoms and discomfort. Scand J Work Environ Health 1985; 11: 457-466.

15. Marcus M, Gerr F. Upper extremity musculoskeletal symptoms among female office workers: associations with video display terminal use and occupational psychosocial stressors. Am J Ind Med 1996; 29: 161-170.

16. Nishiyama K, Nakaseko M, Uehata T. Health aspects of VDT operators in the newspaper industry. In: Grandjean E, ed. Ergonomic and Health in Modern Offices. London: Taylor \& Francis, 1984: 113-118.

17. Rossignol AM, Morse EP, Summers VM, Pagnotto LD. Video display terminal use and reported health symptoms among Massachusetts clerical workers. J Occup Med 1987; 29: 112-118.

18. Sauter SL, Gottlieb MS, Jones KC, Dodson VN, Rohrer KM. Job and health implications of VDT use: initial results of the Wisconsin-NIOSH study. Commun ACM 1983; 26: 284-294.

19. Sauter SL, Schleifer LM, Knutson SJ. Work posture, workstation design, and musculoskeletal discomfort in a VDT data entry task. Hum Factors 1991; 33: 151-167.

20. Smith MJ, Cohen BGF, Stammerjohn LW, Happ A. An investigation of health complaints and job stress in video display operations. Hum Factors 1981; 23: 387-400.

21. Yu ITS, Wong TW. Musculoskeletal problems among VDU workers in a Hong Kong bank. Occup Med 1996; 46: $275-280$.

22. Hagberg M, Wegman DH. Prevalence rates and odds ratios of shoulder-neck diseases in different occupational groups. Br J Ind Med 1987; 44: 602-610.

23. Bergqvist U. Health problems during work with visual display terminals (Thesis). Arbete och Hälsa 1993: 159.

24. Ong C-N, Chia S-E, Jeyaratnam J, Tan K-C. Musculoskeletal disorders among operators of visual display terminals. Scand J Work Environ Health 1995; 21: 60-64.

25. Arndt R. Working posture and musculoskeletal problems of video display terminal operators - review and reappraisal. Am Ind Hyg Assoc J 1983; 44: 437-446.

26. Carter JB, Banister EW. Musculoskeletal problems in VDT work: a review. Ergonomics 1994; 37: 1623-48.

27. Gerr F, Marcus M, Ortiz DJ. Methodological limitations in the study of video display terminal use and upper extremity musculoskeletal disorders. Am J Ind Med 1996; 29: 649-656.

28. Hagberg M, Silverstein B, Wells R, et al. Work related musculoskeletal disorders (WMSDs): A reference book for prevention. London: Taylor \& Francis, 1995: 1-421

29. Musculoskeletal disorders and workplace factors. A critical review of epidemiologic evidence for work-related musculoskeletal disorders of the neck, upper extremity, and low back. NIOSH, 1997.

30. Punnett L, Bergqvist U. Visual display unit work and upper extremity musculoskeletal disorders. A review of epidemiological findings. (National Institute for Working Life - Ergonomic Expert Committee Document No 1). Arbete och Hälsa 1997: 1-161.

31. Sjøgaard G, Sejersted OM, Winkel J, Smolander J, Jørgensen K, Westgaard R. Exposure assessment and mechanisms of pathogenesis in work-related musculoskeletal disorders: Significant aspects in the documentation of risk factors. In: Work and Health. Scientific basis of progress in the working environment. Health and Safety. Report EUR 15980 EN, 1995: 75-87.

32. Viikari-Juntura ERA. The scientific basis for making guidelines and standards to prevent work-related musculoskeletal disorders. Ergonomics 1997; 40: 1097-1117.

33. Rempel D. Musculoskeletal loading and carpal tunnel pressure. In: Gordon SL, Blair SJ, Fine LJ, eds. Repetitive motion disorders of the upper extremity. American academy of orthopaedic surgeons, 1995: 123-132. 